\title{
Nominal Range Error Analysis to support ARAIM
}

\author{
Santiago Perea*o, Michael Meurer*o, Ilaria Martini* and Markus Rippl ${ }^{*}$ \\ *Institute of Communications and Navigation, German Aerospace Center (DLR) \\ ${ }^{\circ}$ Institute of Navigation, RWTH Aachen University, Germany \\ Mathieu Joerger, The University of Arizona \\ Boris Pervan, Illinois Institute of Technology
}

\section{BIOGRAPHY}

Santiago Perea obtained a Master of Science in Aerospace Engineering from University of Sevilla (Spain) in 2013. In parallel, as a double degree student, he gained his Master in Mechanical and Aerospace Engineering from Illinois Institute of Technology (IIT) in 2013. Currently he is part of the staff at the Institute of Communications and Navigation at German Aerospace Center (DLR) and a $\mathrm{PhD}$ candidate at the Institute of Navigation at the RWTH Aachen University. His research focuses on new advanced algorithms for multiconstellation Residual Autonomous Integrity Monitoring (RAIM).

Dr. Michael Meurer received the diploma in electrical engineering and the $\mathrm{PhD}$ degree from the University of Kaiserslautern, Germany. After graduation, he joined the Research Group for Radio Communications at the Technical University of Kaiserslautern, Germany, as a senior key researcher, where he was involved in various international and national projects in the field of communications and navigation both as project coordinator and as technical contributor. From 2003 till 2013, Dr. Meurer was active as a senior lecturer and Associate Professor (PD) at the same university. Since 2006 Dr. Meurer is with the German Aerospace Centre (DLR), Institute of Communications and Navigation, where he is the director of the Department of Navigation and of the center of excellence for satellite navigation. In addition, since 2013 he is a professor of electrical engineering and director of the Institute of Navigation at the RWTH Aachen University. His current research interests include GNSS signals, GNSS receivers, interference and spoofing mitigation and navigation for safety-critical applications.

Dr. Ilaria Martini received the Master Degree in telecommunication engineering and the Ph.D. in information technology from the University of Florence, Italy. She was at the Galileo Project Office of ESA/ESTEC in 2003, working on the performance of the Galileo Integrity Processing Facility. She was research associate in 2004 at the University of Florence and in 2005 at the the Federal Armed Forces Germany in Munich. In 2006 she joined the navigation department of Ifen $\mathrm{GmbH}$ in Munich. Since 2012 she works as research associate in the Institute of Communication and Navigation at the German Aerospace Center (DLR), Oberpfaffenhofen. Her main area of interest is GNSS Integrity Monitoring.

Markus Rippl received his Diploma in Electrical Engineering and Information Technology from Technische Universitat Munchen (TUM) in 2007. Since then, he has been a research associate with the Institute of Communications and Navigation at the German Aerospace Center (DLR), in Oberpfaffenhofen near Munich. His field of work is the integrity of GNSS-based navigation using receiver-side algorithms.

Dr. Mathieu Joerger obtained a Diplôme d'Ingénieur in Mechatronics from the Ecole Nationale Suprieure des Arts et Industries de Strasbourg, France, in 2002, and a M.S. and a Ph.D. in Mechanical and Aerospace Engineering from the Illinois Institute of Technology (IIT), in 2002 and 2009 respectively. He is the 2009 recipient of the Institute of Navigation (ION) Parkinson award, and the 2014 recipient of the IONs Early Achievement Award. He is currently an assistant professor at The University of Arizona, working on multi-sensor integration, on sequential fault-detection for multi-constellation navigation systems, and on relative and differential receiver autonomous integrity monitoring (RAIM).

Dr. Boris Pervan is a Professor of Mechanical and Aerospace Engineering at IIT, where he conducts research on advanced navigation systems. Prior to joining the faculty at IIT, he was a spacecraft mission analyst at Hughes Aircraft Company (now Boeing) and a postdoctoral research associate at Stanford University. Prof. Pervan received his B.S. from the University of Notre Dame, M.S. from the California Institute of Technology, and Ph.D. from Stanford University. He is an Associate Fellow of the AIAA, a Fellow of the Institute of Navigation (ION), and Editor-in-Chief of the ION journal NAVIGATION. He was the recipient of the IIT Sigma Xi Excellence in University Research Award (2011, 2002), Ralph Barnett Mechanical and Aerospace Dept. Outstanding Teaching Award (2009, 
2002), Mechanical and Aerospace Dept. Excellence in Research Award (2007), University Excellence in Teaching Award (2005), IEEE Aerospace and Electronic Systems Society M. Barry Carlton Award (1999), RTCA William E. Jackson Award (1996), Guggenheim Fellowship (Caltech 1987), and Albert J. Zahm Prize in Aeronautics (Notre Dame 1986).

\section{ABSTRACT}

This paper presents a nominal range error analysis to support ARAIM based on a statistical study of orbit and clock errors observations for Galileo F-NAV Open Service. Nominal range error is characterized by the socalled Signal-in-Space Range Error (SISRE) which is computed by comparing precise reference orbits to broadcast ephemeris for each individual satellite. This work processes Galileo constellation service data from March 2015 to July 2016 accounting for the progressive satellite addition during the constellation expansion. Given the undergoing maturation of the Galileo ground segment along with the pending declaration of the Full Operational Capability, the nominality of the error is assessed by disregarding the $1 \%$ of the worst-case samples. Results will address the differences in the nominal range error performance among Galileo satellites highlighting their clear dependence on the satellite clock error behavior. Furthermore, this work evaluates on a monthly basis the evolution of error performance throughout the monitoring period showing a substantial enhancement in the past months in nominal range error. Results advocate for flexible overbounding values contained in the Integrity Support Message (ISM) that are able adapt throughout the constellation evolution along its deployment (i.e. monthly update rate as proposed by Online Architecture).

\section{INTRODUCTION}

By using Multi-frequency Multi-constellation measurement redundancy, the Advanced Receiver Autonomous Integrity Monitoring (ARAIM) airborne algorithm performs consistency checks that aim to detect and exclude ranging measurements coming from potentially faulty satellites. In GNSS Safety-of-Life (SoL) applications such as aircraft precision approach, navigation safety is guaranteed based on whether or not integrity requirement is fulfilled. In order to evaluate this performance metric, inputs from ground monitoring stations, captured in the Integrity Support Message (ISM), are required [1].

ISM parameters (both online and offline architectures) can be divided into two sets of values : fault probabilities and nominal error parameters. The first set describes the probability of a satellite, $P_{\text {sat }}$, and of constellation, $P_{\text {const }}$, being faulted at a given time. The second set characterizes the Signal-in-Space nominal error; two parameters are broadcasted within the ISM for the users to overbound the nominal pseudorange error: User Range Accuracy (URA), also called Signal-in-Space Accuracy (SISA), and maximum nominal bias $\left(b_{n o m}\right)$. The URA/SISA is a one-sigma estimate which indicates confidence in the integrity of satellites ephemeris and clock prediction [2]. Together with $b_{n o m}$, both parameters are used to create a paired overbound (left and right shifted CDF) of the Signal-in-Space Range Error [3]. Both sets of parameters need to be estimated and monitored by the ANSP by means of the ARAIM ground segment.

GNSS literature grants abundant prior work on satellite orbit and clock error analysis. Several studies have targeted the evaluation of GPS broadcast ephemeris performance during its service history such as [4], [5] and [6]. With a particular focus on SoL aviation integrity, the work presented in [7] provided an extensive analysis on ephemeris and clock major faults for GPS constellation during its service history from 2008 until the end of 2014. Our prior work in [8] introduced a new methodology for analyzing orbit and clock errors over time. This approach addresses the changes in biases and standard deviations by breaking service history down into monthly, quarterly and yearly datasets. As of June 2015, this analysis could only be carried out for GPS constellation due to the limited service data that Galileo constellation offered (less than four months of Galileo service history after its last major ground segment update [9]). The study presented in this paper overcomes that constrain by including 17 months of service data for Galileo constellation, being able to monitor up to 9 available satellites (as of July 2016).

The assessment of Galileo range error needs to be treated with care. The fact that Galileo has not still reached its Full Operational Capability (FOC) along with the pending commitment from the Constellation Service Provider (CSP) on the minimum performance might result in a misjudgment of the current integrity accomplishment. Ephemeris and clock error data show outliers that due to this lack of assurance shall not be representative of the future constellation performance. Consequently, a $1 \%$ worst-case exclusion criterion is applied.

First, this paper provides an overview on Galileo F-NAV Dual Frequency E1/E5a Open Service (used by civil aviation) nominal performance through the analysis of the individual ephemeris and clock error components and their weighted contributions to the range error. Second, Galileo service history is broken down into monthly datasets, exposing the significant enhancement that Galileo orbit and clock errors have undergone during the last year, moving from $\sigma_{S I S R E} \sim 90 \mathrm{~cm}$ to $\sigma_{S I S R E} \sim 50 \mathrm{~cm}$. 
This analysis also includes the considerations contained in the latest release of the Galileo Open Service Signal-inSpace Operational Status Definition Document (Galileo OS OSD) [10] regarding health flags, Age of Ephemeris (AoE) and data validity. This work also addresses the evaluation of the Galileo ranging service availability, examining the percentage in time that a satellite is broadcasting healthy signal over the monitoring period.

\section{ERROR COMPUTATION: SIGNAL-IN-SPACE RANGE ERROR}

Out of the different error sources that affect user positioning accuracy (i.e. residual tropospheric errors, second order ionospheric effects, signal multipath, receiver noise, signal deformation), in the context of this work, this analysis accounts only for satellite ephemeris and clock errors. Range error will be defined as the projection of the three orbit error components and the clock error into the user-satellite Line of Sight (LOS) indicated by $\vec{e}_{L O S}$.

Satellite orbit and clock errors are defined as the deviations of the instantaneous satellite Antenna Phase Center (APC) and clock provided by Broadcast Ephemeris (BCE) from the Precise Reference Orbit (PRO) position and satellite clock bias. For illustrative purposes, the three-dimensional orbit error vector will be expressed in satellites's orbit frame Radial, Along-Track and Cross-Track (RAC).

As discussed in [8], for a given instantaneous satellite ephemeris and clock error $\left(\vec{\varepsilon}_{s v, o r b}\right.$ and $\left.\varepsilon_{c l o c k}\right)$, every user $i$ located at distinct points under satellite's footprint will observe a different Instantaneous User Range Error (IURE) defined as

$$
I U R E_{o r b, i}=\vec{\varepsilon}_{s v, o r b}^{T} \cdot \vec{e}_{L O S, i}-\varepsilon_{\text {clock }} .
$$

For integrity purposes, Signal-in-Space Range Error (SISRE) accounts for the worst user projection (wup) within satellite's footprint and can be mathematically defined as

$$
S I S R E_{w u p}=\max \left(\left|I U R E_{i}\right|\right) \cdot \operatorname{sgn}\left(I U R E_{i}\right) .
$$

\section{METHODOLOGY}

From all the five Open Services that Galileo system offers, this work focuses on the Dual Frequency E1/E5a Open Service since it is the one intended for Civil Aviation and consequently for ARAIM users. As described in the previous sections, in order to analyze Galileo nominal range error, satellites ephemeris and clock errors need to be computed. This section provides a comprehensive overview of the implemented steps in the Galileo error computation covering input data sources, availability declaration and data processing chain. Finally, a discussion on the error nominality assessment and outlier exclusion is provided.

As of July 2016, the following nine Galileo satellites are currently declared available [11]: three In-OrbitValidation satellites (E11/GSAT0101, E12/GSAT0102 and E19/GSAT0103) and six Full-Operational-Capability satellites (E26/GSAT0203, E22/GSAT0204, E24/GSAT0205, E30/GSAT0206, E08/GSAT0208 and E09/GSAT0209). The analysis presented in the paper includes Galileo service data after the most recent ground segment update in March 2015 until July 2016, accounting for the progressive satellite addition during the constellation deployment.

\section{A. Data Source}

Galileo satellite orbit and clock error computation requires two sets of data: broadcast ephemeris files and precise reference orbit. Files containing multi-constellation navigation data are generated on a daily basis by the the German Aerospace Center (DLR) and the Technical University of Munich (TUM) and provided in RINEX 3 format. They are computed using measurements from the Multi-GNSS-Experiment (MGEX) stations and are accessible through its online repository ( $b r d m$ files [12]). Precise ephemeris files containing accurate satellite position and clock bias information in SP3 format are provided by the TUM for Galileo satellites and are also publicly available online (tum files [13]).

Although satellite positions provided in BCE and PRO are expressed in the same coordinate system (EarthCentered Earth-Fixed frame, ECEF), they refer to two different points: precise orbit products provide the ECEF coordinates of satellite's Center of Mass (CoM) while broadcast ephemerides datasets are meant to supply satellite's APC ECEF coordinates along with the satellite bias estimation. Clock solutions provided in PRO datasets are also referred to satellite's APC. However, as discussed in [14], there is no reason to assume that both APCs (the one applied by the Galileo control segment and the one used by the MGEX network) are in fact the same point.

In order to compare the broadcast and reference data, satellite positions and clock biases need to be converted to a common reference. For that purpose, two sets of satellite APC offsets need to be used. Table 1 contains the corresponding offset values for precise reference orbits [15] and for the broadcast ephemeris data [16] for each Galileo satellite block: In-Orbit-Validation (IOV) satellites and FullOperational-Capability (FOC) satellites. Next subsection provides the details regarding the conversion between satellite's APC and CoM. 
Table 1: Galileo satellite antenna phase center offset for MGEX network [15] and for broadcast ephemeris (empirically derived in [16])

\begin{tabular}{|c|c|c|c|c|}
\hline Block & Type & $\mathrm{x}(\mathrm{cm})$ & $\mathrm{y}(\mathrm{cm})$ & $\mathrm{z}(\mathrm{cm})$ \\
\hline Galileo IOV & PRO & -20.0 & 0 & +60.0 \\
& BCE & -20.0 & 0 & +75.0 \\
\hline Galileo FOC & PRO & +15.0 & 0 & +100.0 \\
& BCE & +15.0 & 0 & +75.0 \\
\hline
\end{tabular}

\section{B. Health Evaluation and Age of Ephemeris filtering}

In the characterization of satellite nominal range error, only samples corresponding to healthy-flagged navigation datasets can be included. Galileo health flags determination is addressed in the new issue of the Galileo Open Service Signal in Space Operational Status Definition (Galileo OS OSD) [10] recently published by the European Commission. In this document, Galileo Signal-in-Space Health Status for Dual Frequency Open Service E1/E5a $\left(S I S_{E 1, E 5 a}^{H S}\right)$ is defined as the combination of five individual flags following logic

$$
\begin{aligned}
S I S_{E 1, E 5 a}^{H S}= & S H S_{E 1} \wedge D V S_{E 1} \wedge S H S_{E 5 a} \\
& \wedge D V S_{E 5 a} \wedge S I S A_{E 1, E 5 a}^{n o t N A P A}
\end{aligned}
$$

Signal Health Status and Data Validity Status flags for E1 open service $\left(S H S_{E 1}\right.$ and $\left.D V S_{E 1}\right)$ are contained within the INAV datasets while Signal Health Status and Data Validity Status flags for E5a open service $\left(S H S_{E 5 a}\right.$ and $D V S_{E 5 a}$ ) are provided within FNAV datasets [17]. $S I S A_{E 1, E 5 a}^{\text {notNAPA }}$ (Signal-in-Space Accuracy - Not Accuracy Prediction Available for E1/E5a Open Service) is not an actual broadcasted flag. Conversely, it is a flag determined by the user based on the broadcasted value of $S I S A_{E 1, E 5 a}^{\text {ind }}$ (SISA index). The following logic must be applied in th user data processing algorithm

$$
S I S A_{E 1, E 5 a}^{n o t N A P A}=1 \Longleftrightarrow S I S A_{E 1, E 5 a}^{i n d}=255 .
$$

According to Galileo OS ICD [17], users are not required to perform any check of the navigation data Age of Ephemeris (AoE) since it is satellite on-board software's duty to set DVS flag to true in case of excessive aging of data. However, in order to avoid inconsistencies in the compilation process of the $b r d m$ files, this work implements the following check,

$$
A o E(t) \leq 3 h .
$$

AoE is defined as the elapsed time between the reception of the message $t$ and the time of ephemeris $t_{o e}$ as broadcast by the navigation message [10]

$$
A o E(t)=t-t_{o e} .
$$

In order for a navigation dataset to be used in our error characterization, condition (5) must be fulfilled.

\section{Data Processing Chain}

Figure 1 provides an overview of the steps that need to be implemented in the satellite ephemeris and clock error evaluation. For each given time stamp (sampling rate of 5 minutes from March 6th 2015 until July 31st 2016) the selected BCE dataset is utilized to emulate user's calculation of satellite position and clock. Dataset selection and health evaluation must be done according to (3) and (5) conditions.

In order to compare estimated satellite positions and clock bias to the precise reference products, they must be translated to satellite CoM using the corresponding APC offsets provided in Table 1. Given that these offsets are given is satellite's body-fixed (BF) frame while BCE and PRO position and clock data are provided in ECEF, it is necessary to appeal to spacecraft dynamics theory to carry out this transformation. By implementing Galileo satellite attitude model detailed in [18], both broadcast and precise orbit and clock data are commuted to a common reference, satellite's CoM. The resulting discordance between these two inputs is the so-called orbit and clock error vector.

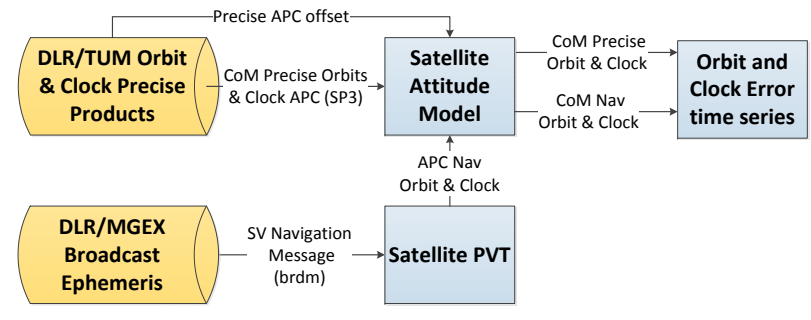

Figure 1: Galileo ephemeris and clock error data processing chain

\section{Nominality assessment}

This work's main scope is the assessment of Galileo nominal range error to provide a set of recommendations to the ISM generation. When speaking about Galileo range errors, several facts need to be clearly stated. Galileo deployment is still an ongoing process full of continuous upgrades not only in the ground but also in the space segment. The Galileo system gradually matures as satellites are added to the constellation. On top of that, Galileo operational duties have not been assigned to any Constellation Service Provider yet, meaning that no commitments on minimum performance have been made.

Ephemeris and clock error data show large outliers that, due to this lack of assurance, might lead to a misjudgment of the current integrity accomplishment. In fact those large errors shall not be representative of the future constellation performance once the CSP has established a minimum performance commitment. Since this paper is addressing the 
nominal error characterization, it is legit to exclude the $1 \%$ of the worst-case samples from the error distributions. In order to conduct a fair comparison, this exclusion is individually applied to each satellite. This work does not aim to determine $P_{\text {sat }}$ neither $P_{\text {const }}$; future revisions of this study will address Galileo satellites and constellation failure rate once the minimum operational performance commitments are set.

\section{GALILEO CONSTELLATION SERVICE HIS- TORY OVERVIEW: DISTRIBUTION AND CORRELATION}

This section pursues a statistical characterization of the Galileo nominal range error based on the study of satellite orbit and clock during the 17 month monitoring period. The first subsection addresses the per-satellite availability of the Dual Frequency E1/E5a service along with an analysis of the number of excluded samples. Second subsection provides the distribution statistics for each individual error component (Radial, Along-Track, Cross-Track and Clock error) along with a correlation versus SISRE analysis.

\section{A. Sample size and Exclusions}

Galileo service data from March 2015 to July 2016 has been processed applying the technique detailed in sections II and III. The study included 514 days of monitoring providing a total of 705,732 healthy samples after $1 \%$ worstcase exclusion. For each one of the nine analyzed satellites, Table 2 details the service availability defined as the percentage of time that a satellite is transmitting healthy signals for the E1/E5a service since it was declared available (or March 2015 for IOV satellites) until July 31st. The second column of Table 2 contains the number of healthy samples that remain after the exclusion criterion applied to each satellite.

Table 2: Galileo Open Service Dual Frequency E1/E5a Service Availability from March 6, 2016 to July 31, 2016

\begin{tabular}{|c|c|c|}
\hline $\begin{array}{c}\text { Galileo } \\
\text { Satellite ID }\end{array}$ & $\begin{array}{c}\text { Health } \\
\text { Availability }(\%)\end{array}$ & $\begin{array}{c}\text { Healthy Samples } \\
\text { after exclusion }\end{array}$ \\
\hline E08/FOC0208 & 99.31 & 28,597 \\
\hline E09/FOC0209 & 99.31 & 28,598 \\
\hline E11/IOV0101 & 93.06 & 136,388 \\
\hline E12/IOV0102 & 95.83 & 140,439 \\
\hline E19/IOV0103 & 93.55 & 137,104 \\
\hline E22/FOC0204 & 93.25 & 64,074 \\
\hline E24/FOC0205 & 93.39 & 49,527 \\
\hline E26/FOC0203 & 98.69 & 68,118 \\
\hline E30/FOC0206 & 99.73 & 52,887 \\
\hline
\end{tabular}

Figure 2 includes E1/E5a service time line starting on the availability declaration date for FOC satellites and on
March 6th 2015 for IOV satellites. Samples corresponding to healthy datasets are represented in green, blue for samples from unhealthy periods and red for those samples belonging to the excluded $1 \%$ worst-case.

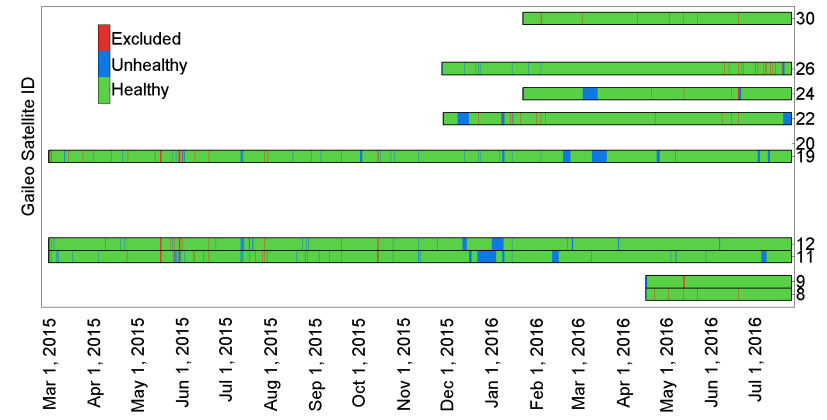

Figure 2: Galileo constellation service history time line

\section{B. Nominal Error distribution and correlation}

Ephemeris and clock error statistical results are displayed in four different formats: Relative Frequency Histograms (RHF), Folded Cumulative Distribution Functions (FCDF) [19], scatter plots and table of statistics. Figures 3 and 4 respectively represent RHF and FCDF for Galileo constellation during the 17 months of observation (merging the 705,732 samples coming from the nine monitored satellites). As occurs for GPS orbit errors [8], along-track component presents the largest error magnitude and dispersion, fact that can be ascribed to the low observability that the orbit determination process has in that direction. In terms of magnitude, cross-track error is next. Although not shown here, this component presents a particular half sidereal day periodicity which is attributable to the harmonic components of the satellite's equation of motion [20]. Respectively, radial component displays the smallest error magnitude and dispersion.

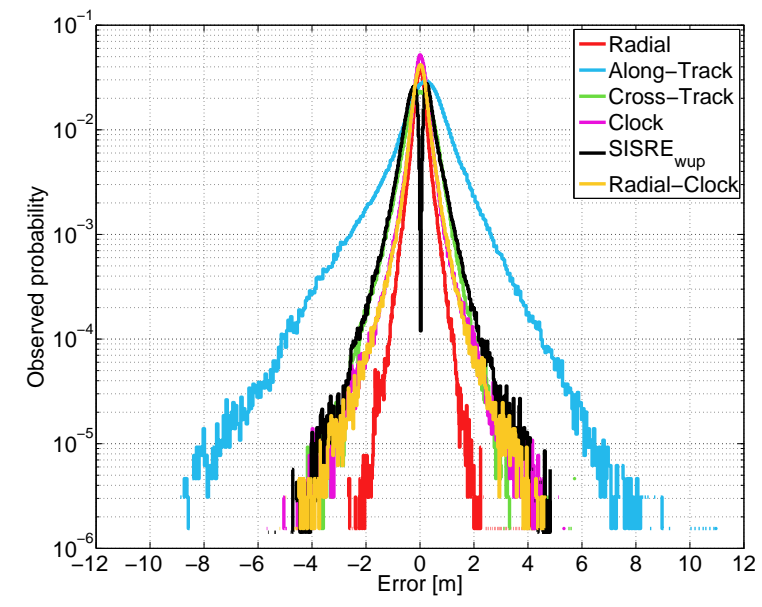

Figure 3: Galileo orbit and clock error Relative Frequency Histogram from 3/6/2015 to 7/31/2016 


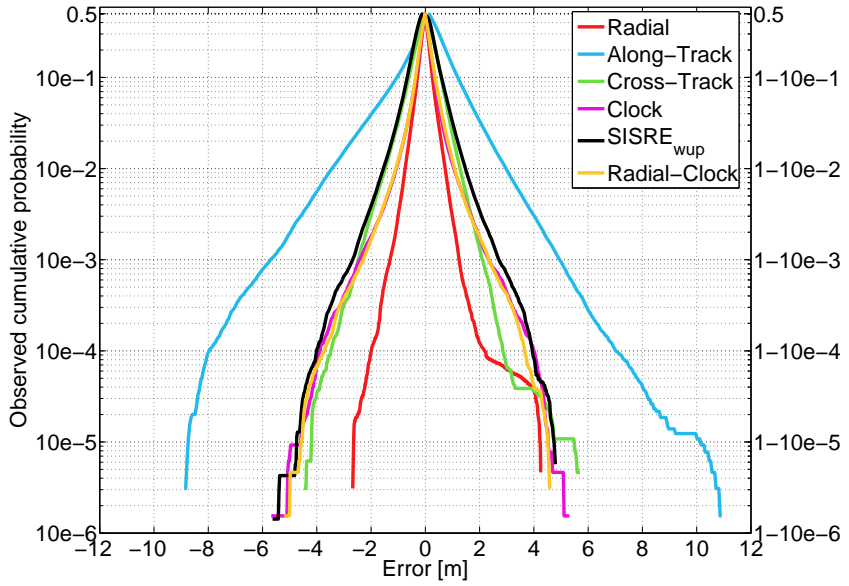

Figure 4: Galileo orbit and clock error FCDF from $3 / 6 / 2015$ to $7 / 31 / 2016$

RFH and FCDF plots also include SISRE wup $_{\text {, clock and }}$ radial-minus-clock error distributions. As reflected in (1), clock error component directly translates into the range error. In addition, range error also receives almost the total magnitude of the radial error (weighting factor between 0.977 and 1). However, range error is dominated by the clock component given its larger magnitude w.r.t. radial error as displayed in Figure 4. It can also be seen that radial-minus-clock error and SISRE $_{\text {wup }}$ distributions display matching FCDF, especially in low probabilities.

Table 3 allows the assessment of the prior statements for each individual satellite under study. Each row provides samples mean $(\mu)$ and standard deviation $(\sigma)$ for radial, along-track, cross-track, clock and radial-minus-clock error distributions along with standard deviation for wup range error $\sigma_{S I S R E}$. Given its definition in (2), SISRE wup cannot present zero values, fact that entails an inherent bi-modality of the error distribution centered around the origin (Figure 3).

Scatter plots in Figure 5 evaluate the correlation between SISRE $_{\text {wup }}$ and each individual error component. Each plot respectively contains the covariance value $\left(\sigma_{x, y}^{2}\right.$ in $\left.m \cdot m\right)$ between radial, along-track, cross-track and clock error and SISRE $_{\text {wup }}$ together with the correlation coefficient defined as $\rho_{x, y}=\sigma_{x, y} /\left(\sigma_{x} \cdot \sigma_{y}\right)$ in [21]. Correlation coefficient $\rho_{\text {rad,sisre }}=0.391$ and $\rho_{\text {clk,sisre }}=-0.664$ corroborates the prior assertions on $\mathrm{SISRE}_{\text {wup }}$ dependence on clock and radial error. As depicted in Table 3 and Figures 3, 4 and 5, although along-track error presents large magnitudes compared to the rest of components its contribution to range error is the most limited one (maximum weighting factor of 0.214 )
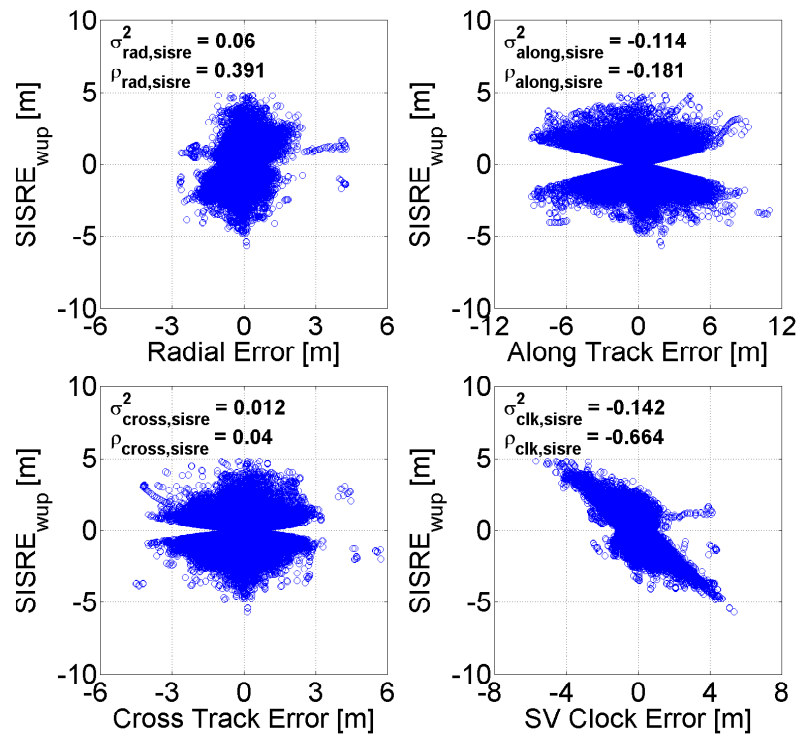

Figure 5: Scatter plot Galileo orbit and clock error versus SISRE $_{\text {wup }}$ from $3 / 6 / 2015$ to $7 / 31 / 2016\left(\sigma_{x, y}^{2}\right.$ in $\left.m \cdot m\right)$

In order to compare the nominal error performance among satellites, Figure 6 presents clock error FCDF for the nine monitored Galileo space vehicles. As depicted in the plot, satellites that display lower clock errors dispersion and magnitude also show lower $\sigma_{S I S R E}$ and vice versa. For example, E08 and E22 show the smallest error magnitudes in Figure 6 while E19 and E26 the largest ones. Looking at Table 3, the following reciprocity can be found:

$$
\begin{gathered}
\sigma_{c l k}^{E 08}<\sigma_{c l k}^{E 22} \ll \sigma_{c l k}^{E 19} \\
\sigma_{S I S R E}^{E 08}<\sigma_{S I S R E}^{E 22} \ll \sigma_{S I S R E}^{E 19} .
\end{gathered}
$$

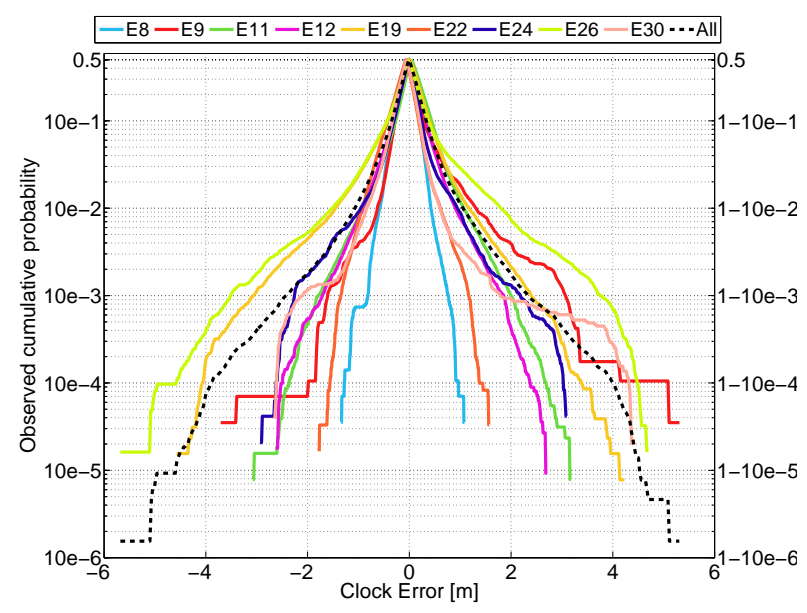

Figure 6: Galileo clock error comparison - FCDF from 3/6/2015 to 7/31/2016 
Table 3: Galileo ephemeris and clock error statistics by satellite from 3/6/2015 to 7/31/2016 (values in cm)

\begin{tabular}{|c||c|c||c|c||c|c||c|c||c|c||c|}
\hline \multirow{2}{*}{$\begin{array}{c}\text { Galileo } \\
\text { Satellite ID }\end{array}$} & \multicolumn{2}{c|}{ Radial } & \multicolumn{2}{c|}{ Along-Track } & \multicolumn{2}{c|}{ Cross-Track } & \multicolumn{2}{c|}{ Clock } & \multicolumn{2}{c|}{ Radial-Clock } & SISRE $_{\text {wup }}$ \\
\cline { 2 - 13 } & $\mu$ & $\sigma$ & $\mu$ & $\sigma$ & $\mu$ & $\sigma$ & $\mu$ & $\sigma$ & $\mu$ & $\sigma$ & $\sigma$ \\
\hline \hline E08/FOC0208 & -0.3 & 15.3 & 22.8 & 66.9 & 3.0 & 30.3 & -4.8 & 20.8 & 4.5 & 17.6 & 30.2 \\
\hline E09/FOC0209 & -0.1 & 17.3 & 33.6 & 72.4 & 2.7 & 31.0 & 1.0 & 34.4 & -1.0 & 32.8 & 45.4 \\
\hline E11/IOV0101 & -1.6 & 32.0 & 3.6 & 124.7 & -0.6 & 57.6 & 6.25 & 34.3 & -7.9 & 37.6 & 57.6 \\
\hline E12/IOV0102 & -3.6 & 29.9 & -2.2 & 114.0 & -0.8 & 60.7 & 0.5 & 31.7 & -4.1 & 35.9 & 58.5 \\
\hline E19/IOV0103 & -2.8 & 30.8 & -11.9 & 143.6 & -0.6 & 58.8 & -1.0 & 44.3 & -2.0 & 45.5 & 69.1 \\
\hline E22/FOC0204 & -0.3 & 18.4 & 11.8 & 69.6 & 1.0 & 37.9 & -9.5 & 27.4 & 9.3 & 23.2 & 34.7 \\
\hline E24/FOC0205 & 2.7 & 15.9 & 37.6 & 68.0 & -1.3 & 33.9 & -1.8 & 31.2 & 4.6 & 31.3 & 43.4 \\
\hline E26/FOC0203 & -0.4 & 22.1 & 29.1 & 78.7 & 1.3 & 43.6 & -1.5 & 52.3 & 1.1 & 53.0 & 63.4 \\
\hline E30/FOC0206 & 0.5 & 22.4 & 23.9 & 72.6 & -1.3 & 36.2 & -6.3 & 28.1 & 6.8 & 23.6 & 35.2 \\
\hline Constellation & -1.3 & 26.3 & 9.1 & 109.2 & -1.2 & 52.0 & -0.6 & 36.9 & -0.7 & 37.9 & 57.9 \\
\hline
\end{tabular}

\section{GALILEO ORBIT AND CLOCK ERROR: EVO- LUTION OVER MONITORING PERIOD}

The previous section has provided an overview on Galileo F-NAV Dual Frequency E1/E5a Open Service nominal range error performance through the analysis of the individual error components. If an ISM was to be disseminated based on the observed service data, considerations included in (7) and (8) would be determining in the nominal range error overbound. The main premise of the prior section is the assumption of the stationarity of the error. However, the characterization of satellite ephemeris and clock error by merging all the service history data in a single distribution does not provide enough information about the evolution of the error. In case of young constellations still under deployment, error evolution analysis can be decisive; this section addresses the results of this approach.

By breaking service data down into monthly datasets, Figure 7 displays the corresponding value of SISRE $_{\text {wup }}$ standard deviation for each individual satellite. The most remarkable time-dependent effect in the plot is the significant reduction in $\sigma_{S I S R E}$ that FOC satellites experienced from March-April 2015 until December 2015. Range error standard deviation was reduced in more than the 50\% throughout the past year. By the time the first two FOC satellite became available, $\sigma_{S I S R E}$ had reached values below $50 \mathrm{~cm}$ for IOV satellites. This enhancement in the performance shall be attributed to the inherent maturation of the constellation which presumably entailed an enhancement in the orbit determination and time synchronization process.

From Table 3 we extracted that standard deviation for E19 range error presented a quite larger value than in the case of E08 and E22 satellites. It is true that if the full service history is merged that statement is accurate. However, Figure 7 shows that by the time E22 was declared available, $\sigma_{S I S R E}^{E 22}$ and $\sigma_{S I S R E}^{E 19}$ had close values (standing between
40-45 cm). In fact they maintained the same order of magnitude during the rest of the monitoring period. That can be extrapolated to the rest of the IOV. It is here where the time-dependence analysis plays an essential role. Due to the continuous change in the Galileo system, satellites performance can only be compared in the same time frame.

In order to check whether users were informed of this improvement in the orbit and clock estimation accuracy, Figure 8 represents the standard deviation values of the range error normalized by the broadcast SISA. No significant change is observed in the shape of the curve, meaning that SISA values did not reflect the enhancement in the range accuracy.

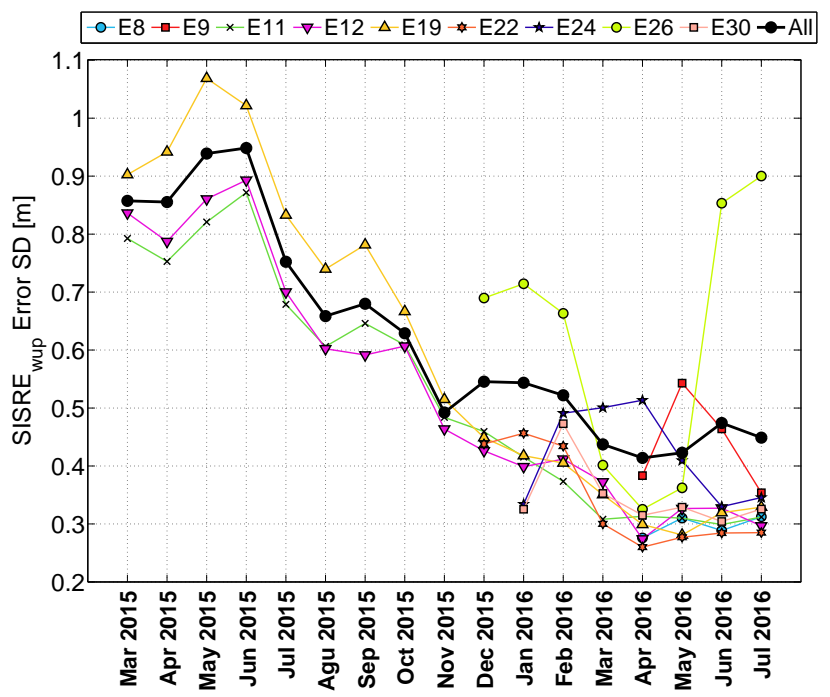

Figure 7: Galileo SISRE $_{\text {wup }}$ monthly standard deviation from March 2015 to July 2016

Figure 9 includes a similar analysis. For each satellite, it plots the monthly standard deviation value for radial, along-track, cross-track and clock error distributions. An analogous interpretation can be read; the enhancement of 


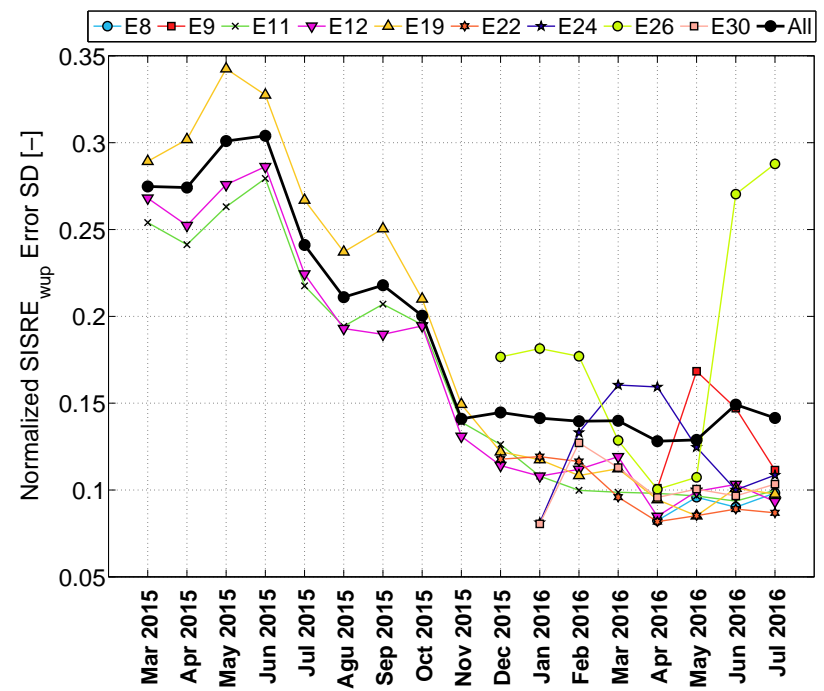

Figure 8: Galileo SISRE $_{\text {wup }}$ monthly standard deviation normalized by broadcast SISA from March 2015 to July 2016

orbit and clock error entailed a decreased over the 50\% in $\sigma$ values. The particular case of E26 is worth to mention. In the last two months of the observation period, satellite E26 clock error has presented anomalously large magnitudes. Although it showed values quite similar to the rest of the constellation during March-May 2016 (around $30 \mathrm{~cm}$ ), errors during the last two months of the observation period were surprisingly large (Figure 9). It has been observed that this behavior was just temporal and ended by mid of July. After an unhealthy period of almost 2 days (Figure 2), on July 21st 2016 satellite performance came back to levels of April. This temporal variation in satellite E26 clock error is another fact to support the relevance of the time-adaptive overbound.

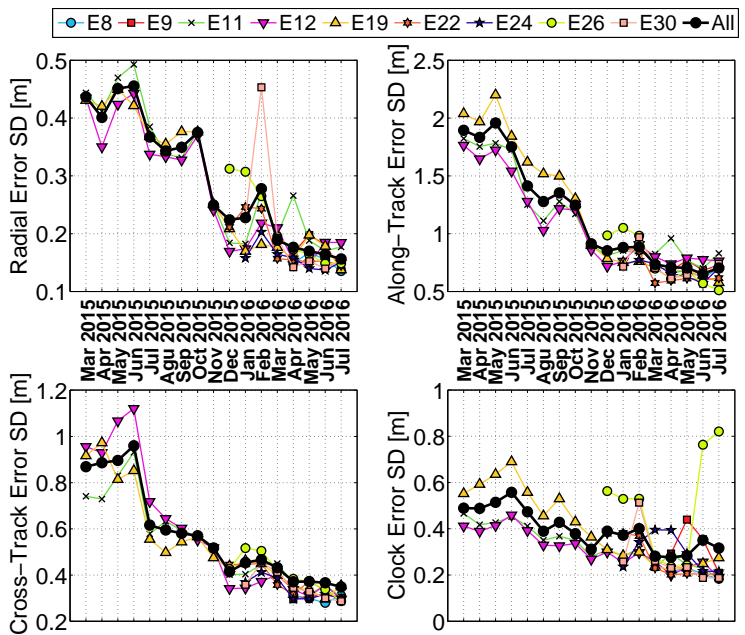

Figure 9: Galileo Radial, Along-Track, Cross-Track and Clock error monthly standard deviation from March 2015 to July 2016
In order to illustrate the time-variant component of the error distribution, IOV satellite E19 is used as example. Waterfall plots in Figures 10, 11 and 12 represent the monthly evolution of radial, clock and SISRE $_{\text {wup }}$ error FCDF throughout the monitoring period. The substantial reduction from March to December 2015 in error magnitude is visible in both radial and clock error implying a performance enhancement of the range error. Note that abscissa axis in Figure 11 has been inverted in order to see the influence of large clock errors into the SISRE $E_{\text {wup }}$ (Figure 12). It is also interesting to remark that Galileo radial and clock errors do not present a significant bias variation over months like in the GPS case where monthly variations of over $15 \mathrm{~cm}$ have been observed [8].

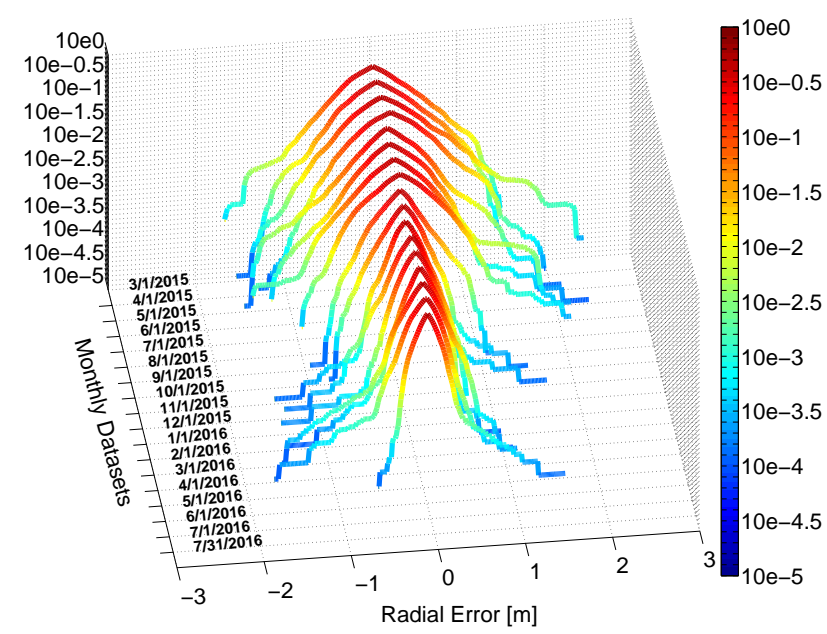

Figure 10: Galileo GSAT0103/PRN19 radial error FCDF waterfall monthly from $3 / 6 / 2015$ to $7 / 31 / 2016$

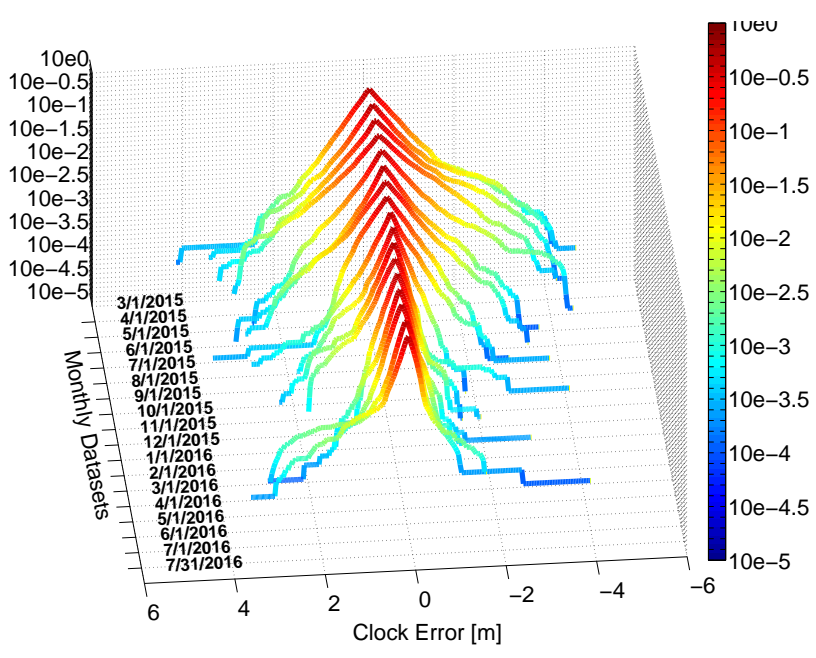

Figure 11: Galileo GSAT0103/PRN19 clock error FCDF waterfall monthly from $3 / 6 / 2015$ to $7 / 31 / 2016$ 


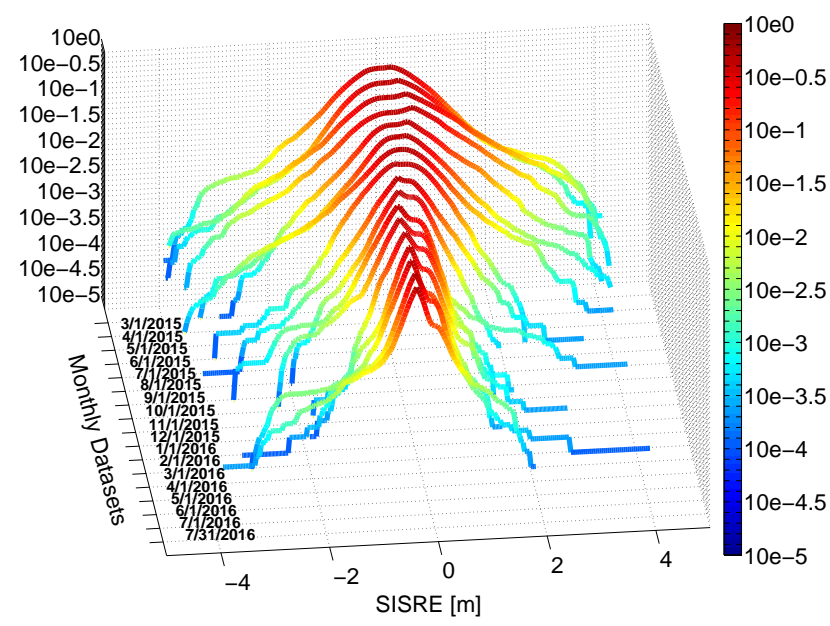

Figure 12: Galileo GSAT0103/PRN19 SISRE wup $_{\text {FCDF }}$ waterfall monthly from $3 / 6 / 2015$ to $7 / 31 / 2016$

As previously done in the error standard deviation analysis, SISRE $_{\text {wup }}$ is normalized by the broadcast SISA. An analogous conclusion can be extracted in this case; SISA values did not reflect the improvement in the estimation accuracy of satellite E19. This assertion is supported by the fact that both absolute and normalized SISRE wup $_{\text {FCDF (Figures } 12}$ and 13 respectively) present the same relative monthly variations.

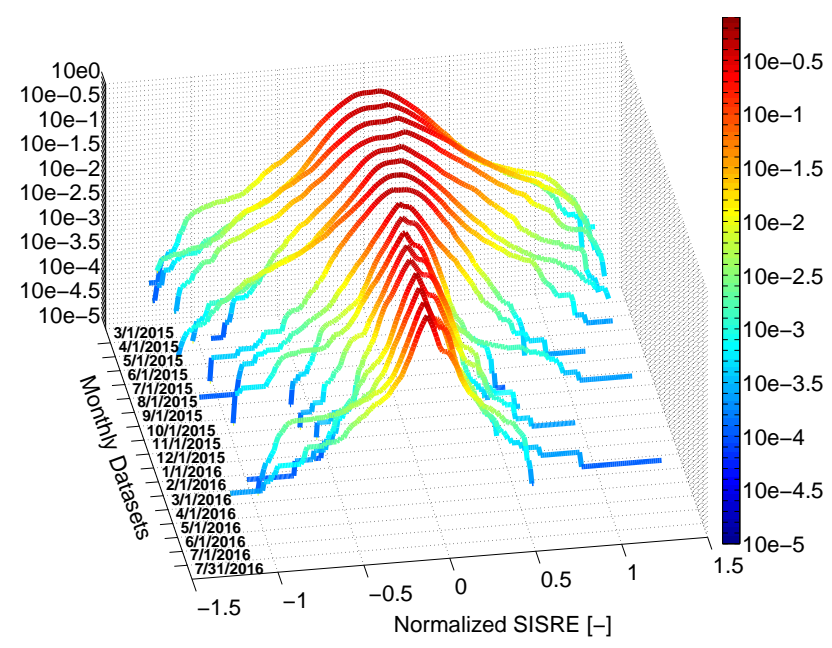

Figure 13: Galileo GSAT0103/PRN19 normalized clock error FCDF waterfall monthly from 3/6/2015 to 7/31/2016

\section{CONCLUSIONS}

The work presented in this paper has characterized Galileo satellites nominal range error throughout the last 17 months of service. Contrasting results have been found in the satellites ranging errors depending on the time period under analysis. Time evolution study has shown that Galileo constellation performance significantly enhanced from March to December 2015 where a reduction of more than the $50 \%$ in $\sigma_{\text {SISRE }}$ was observed in the FOC satellites. This variations could presumably be attributable to the maturation of the system through satellites deployment.

Time evolution plays an essential role in the ISM parameters evaluation. Results have shown that merging all the available data in a single set might lead to over conservative judgment of the satellite's nominal performance. Consequently, ISM parameters computation is recommended to account for this variability in time. Instead of hard-coded values, results advocate for flexible overbounding parameters that are able adapt throughout the constellation evolution (i.e. monthly update rate as proposed by Online Architecture).

As mentioned earlier in this paper, the fact that Galileo has not still reached its full operational capability along with the lack of commitment from a designated service provider has motivated the exclusion of the $1 \%$ worst-worst case samples. Future revisions of this work will deepen in the study of Galileo anomalies, assessing not only the nominal performance but also the fault magnitude and rates.

\section{ACKNOWLEDGMENTS}

The authors would like to deeply thank Dr. Oliver Montenbruck and Dr. Peter Steigenberger from the German Space Operation Center (GSOC) for their support and advice in the elaboration of this work.

\section{REFERENCES}

[1] Working Group C, ARAIM Technical Subgroup, EUUS Cooperation in Satellite Navigation, "Milestone 3 report," Tech. Rep., March 2016.

[2] Global Positioning System Standard Positioning Service Performance Standard, U.S. Department of Defence, 4th Ed. September 2008, pp A-22, Std.

[3] J. Rife, S. Pullen, P. Enge, and B. Pervan, "Paired Overbounding for Nonideal LAAS and WAAS Error Distributions," IEEE Tansactions on Aerospace and Electronic Systems, vol. 42, pp. 1386-1395, 2006.

[4] D. L. M. Warren and J. F. Raquet, "Broadcast vs. precise gps ephemerides: a historical perspective," GPS Solutions, vol. 7, no. 3, 2003. [Online]. Available: http://dx.doi.org/10.1007/s10291-003-0065-3

[5] C. Cohenour and F. Van Graas, "GPS Orbit and Clock Error Distributions," NAVIGATION, vol. 58, no. 1, Spring 2011.

[6] T. Walter, J. Blanch, and P. Enge, "Evaluation of Signal in Space Error Bounds to Support Aviation Integrity," NAVIGATION, vol. 57, no. 2, pp. 101-113, Summer 2010. 
[7] T. Walter and J. Blanch, "Charactrization of GPS Clock and Ephemeris Errors to support ARAIM," in Pacific PNT Meeting, Honolulu, HI, April 20-23.

[8] S. Perea, M. Meurer, M. Rippl, B. Belabbas, M. Joerger, and B. Pervan, "URA/SISA Analysis for GPSGalileo ARAIM Integrity Support Message," in Proceedings of the 28th International Technical Meeting of The Satellite Division of the Institute of Navigation (ION GNSS+ 2015), Tampa, Florida, September 2015, pp. 735-745.

[9] D. Blonski, "GALILEO System Status," in Proceedings of the 28th International Technical Meeting of the Satellite Division of the Institute of Navigation (ION GNSS+ 2015), Tampa, FL, September 2015, pp. 1241-1263.

[10] European Comission, Galileo Open Service Signalin-Space Operational Status Definition, Issue 1.1, Std., July 2016.

[11] European GNSS Service Center, Galileo Constellation Information, "https://www.gsceuropa.eu/system-status/constellation-information."

[12] Multi-GNSS Experiment Repository, $\mathrm{ftp}: / / c d d i s . g s f c . n a s a . g o v / g n s s / d a t a / c a m p a i g n / m g e x$.

[13] Technical University of Munich / MGEX Repository, ftp://cddis.gsfc.nasa.gov/pub/gps.

[14] O. Montenbruck, P. Steigenberger, and A. Hauschild, "Broadcast versus precise ephemeris: a multi-gnss perspective," GPS Solutions, June 2014.

[15] MGEX, Galielo Antenna Phase Center Offsets, http://mgex.igs.org/IGS 'MGEX'Status 'GAL.html.

[16] P. Steigenberger, M. Fritsche, R. Dach, R. Schmid, O. Montenbruck, M. Uhlemann, and L. Prange, "Estimation of satellite antenna phase center offsets for Galileo," Journal of Geodesy, vol. 90, no. 8, pp. 773785, August 2016.

[17] Galileo Signal in Space Interface Control Document, Issue 1.2, European Comission Std., November 2015.

[18] O. Montenbruck, R. Schmid, F. Mercier, P. Steigenberger, C. Noll, R. Fatkulin, S. Kogure, and A. Ganeshan, "GNSS satellite geometry and attitude models," Advances in Space Research, vol. 56, no. 6, pp. 10151029, September 2015.

[19] K. L. Monti, "Folded Empirical Distribution Function Curves-Mountain Plots," The American Statistician, vol. 49, no. 4, pp. 342-345, November 1995.

[20] O. Montenbruck and E. Gill, Satellite Orbits, 3rd ed. Springer-Verlag, 2005.

[21] D. C. Montgomery and G. C. Runger, Applied Statistics and Probability for Engineers, 3rd ed. John Wiley \& Sons, Sept 2002. 